BMJ Open

Diabetes

Research

\& Care

\title{
Predictors of arterial stiffness in adolescents and adults with type 1 diabetes: a cross-sectional study
}

\author{
Kaitlin M Love (D , , William B Horton, ${ }^{1}$ James T Patrie, ${ }^{2}$ Linda A Jahn, \\ Lee M Hartline, ${ }^{1}$ Eugene J Barrett ${ }^{1,3}$
}

To cite: Love KM, Horton WB, Patrie JT, et al. Predictors of arterial stiffness in adolescents and adults with type 1 diabetes: a cross-sectional study. BMJ Open Diab Res Care 2022;10:e002491. doi:10.1136/ bmjdrc-2021-002491

- Additional supplemental material is published online only. To view, please visit the journal online (http://dx.doi. org/10.1136/bmjdrc-2021002491).

KML and WBH are joint first authors.

Received 19 July 2021

Accepted 10 December 2021

Check for updates

(c) Author(s) (or their employer(s)) 2022. Re-use permitted under CC BY-NC. No commercial re-use. See rights and permissions. Published by BMJ.

${ }^{1}$ Division of Endocrinology and Metabolism, Department of Medicine, University of Virginia Health System, Charlottesville Virginia, USA

${ }^{2}$ Department of Public Health Sciences, University of Virginia Health System, Charlottesville, Virginia, USA

${ }^{3}$ Department of Pharmacology, University of Virginia Health System, Charlottesville, Virginia, USA

Correspondence to Dr Kaitlin M Love; kml2w@virginia.edu

\section{ABSTRACT}

Introduction Individuals with type 1 diabetes have increased arterial stiffness compared with age-matched healthy controls. Our aim was to determine which hemodynamic and demographic factors predict arterial stiffness in this population.

Research design and methods Carotid-femoral pulse wave velocity (cfPWV) was examined in 41 young adults and adolescents with type 1 diabetes without microvascular complications. Two ordinary least squares regression analyses were performed to determine multivariate relationships between cfPWV $\left(\log _{\mathrm{e}}\right)$ and (1) age, duration of diabetes, sex, and hemoglobin A1c and (2) augmentation index (Alx), mean arterial pressure, flowmediated dilation (FMD), and heart rate. We also examined differences in macrovascular outcome measures between sexes.

Results Age, sex, and FMD provided unique predictive information about cfPWV in these participants with type 1 diabetes. Despite having similar cardiovascular risk factors, men had higher cfPWV compared with women but no differences were observed in other macrovascular outcomes (including FMD and Alx).

Conclusions Only age, sex, and FMD were uniquely associated with arterial stiffness in adolescents and adults with uncomplicated type 1 diabetes. Women had less arterial stiffness and similar nitric oxide-dependent endothelial function compared with men. Larger, prospective investigation is warranted to determine the temporal order of and sex differences in arterial dysfunction in type 1 diabetes.

\section{INTRODUCTION}

Large elastic arteries stiffen with accrual of age and other cardiovascular risk factors. This process holds a number of deleterious consequences for the cardiovascular system and major organs, as arterial stiffness is an independent determinant of cardiovascular disease (CVD) risk. ${ }^{1}$ Diabetes mellitus (DM) is a cardiovascular risk factor intricately linked to arterial stiffness given that: (1) arterial stiffness is influenced by both hemodynamic forces and extrinsic factors (including hormones, salt, and glucose) $;^{2}$ (2) arterial stiffness increases with deteriorating glucose tolerance status ${ }^{3}$ and is associated with insulin

\section{Significance of this study}

What is already known about this subject?

- A measure of central artery stiffness, carotidfemoral pulse wave velocity (cfPWV) predicts renal outcomes, cardiovascular events, and mortality in persons with type 1 diabetes.

- Age, race, mean arterial pressure, waist-to-height ratio/body mass index, presence of microalbuminuria have all been uniquely associated with cfPWV in populations with type 1 diabetes.

- The relationship between cfPWV and flow-mediated dilation (FMD), a measure of nitric oxide (NO)dependent endothelial function at the brachial artery, has not been defined in type 1 diabetes, and the relationship between diabetes duration and these vascular measures are unclear.

What are the new findings?

- Arterial stiffness and NO-dependent endothelial dysfunction were highly prevalent even within 5 years of diabetes diagnosis ( $16.7 \%$ and $83.3 \%$ respectively) in this cohort of adolescents and adults with uncomplicated type 1 diabetes.

- FMD was uniquely associated with cfPWV.

- Augmentation index (Alx), mean arterial pressure, and diabetes duration were not predictive of cfPWV.

- Despite a greater excess cardiovascular risk associated with type 1 diabetes in women compared with men, macrovascular function was no worse in women when examining sex differences.

- Women had a lower cfPWV and similar Alx and FMD compared with men.

How might these results change the focus of research or clinical practice?

- Larger, prospective clinical investigation is needed in type 1 diabetes to determine the temporal order of and sex differences in arterial dysfunction.

resistance $^{4}$ and (3) arterial stiffness is acutely increased during postprandial hyperglycemia in patients with type $2 \mathrm{DM}^{5}$

People with type $1 \mathrm{DM}$ have significantly increased arterial stiffness, assessed by carotidfemoral pulse wave velocity ${ }^{6}$ (cfPWV) or brachial-ankle pulse wave velocity, ${ }^{7}$ compared 
with age-matched and sex-matched healthy controls, and this increase occurs independent of traditional cardiovascular risk factors. ${ }^{6}$ Notably, these changes occur early in the course of disease, as adolescents with type $1 \mathrm{DM}$ exhibit increased arterial stiffness compared with healthy controls. ${ }^{8-11}$ Recent prospective cohort studies have also shown that markers of arterial stiffness, including cfPWV and radial artery augmentation index (AIx), predict renal outcomes, cardiovascular events, and mortality in type $1 \mathrm{DM}^{12}{ }^{13}$ Despite the well-established relationship between arterial stiffness and type $1 \mathrm{DM}$, few studies have attempted to define the specific factors associated with elevated arterial stiffness in this population. ${ }^{6}{ }^{14}$ To our knowledge, no studies have examined the relationship between brachial artery flow-mediated dilation (FMD; a marker of vascular nitric oxide (NO)-dependent endothelial function) and cfPWV in type $1 \mathrm{DM}$.

In the current study, we endeavored to clarify the determinants of increased cfPWV in individuals with type 1 DM. Our prespecified hypothesis was that, among demographic and vascular parameters, duration of DM, mean arterial pressure, and AIx would provide unique predictive information about cfPWV in individuals with type 1 DM. We also hypothesized that men and women with type $1 \mathrm{DM}$ would have comparable markers of arterial stiffness (ie, cfPWV and AIx) and vascular endothelial function (ie, FMD).

\section{RESEARCH DESIGN AND METHODS}

\section{Recruitment and study population}

Following institutional review board approval, we recruited both an adult and an adolescent cohort by public advertisement and direct mailings. An initial telephone interview was conducted with all respondents, and eligible study participants then fasted overnight and presented to the University of Virginia (UVA) Clinical Research Unit (CRU) for a screening visit.

\section{Clinical assessment and initial screening}

All screening visits and study protocols were conducted at the UVA CRU. Screening included a detailed medical history and physical examination along with fasting measures of complete blood count, comprehensive metabolic panel, lipid panel, hemoglobin A1c (HbAlc), C-peptide, serum pregnancy test, and urine for albumin/ creatinine ratio. Adults with type $1 \mathrm{DM}$ met inclusion criteria if they were $\geq 18$ and $\leq 50$ years old, had body mass index (BMI) $<30 \mathrm{~kg} / \mathrm{m}^{2}$, had HbAlc $<9.0 \%(<75 \mathrm{mmol} /$ $\mathrm{mol}$ ), and had blood pressure $<160 / 90 \mathrm{~mm} \mathrm{Hg}$ at time of screening. The diagnosis of type $1 \mathrm{DM}$ was based on: (1) history of initial presentation with symptomatic hyperglycemia, polyuria, with or without ketonemia, in individuals $<25$ years of age, of normal body weight (BMI $<25 \mathrm{~kg}$ / $\mathrm{m}^{2}$ ) who required insulin treatment from the initial diagnosis of diabetes and (2) fasting C-peptide $<0.2 \mathrm{nmol} / \mathrm{L}$ in participants with a 5-year or greater history of diabetes. ${ }^{15} 16$ Potential participants were excluded from the adult cohort if they were current smokers or had quit smoking $<6$ months prior, were taking vasoactive medications (eg, diuretics, statins, etc) outside of a stable dose of antihypertensive medication, were pregnant (ie, positive pregnancy test) or nursing, had any known prior microvascular complications due to type $1 \mathrm{DM}$, had history of cardiovascular, peripheral vascular, or liver disease, had history of ketoacidosis within the previous 12 calendar months, had low-density lipoprotein (LDL) cholesterol $\geq 160 \mathrm{mg} / \mathrm{dL}(4.1 \mathrm{mmol} / \mathrm{L})$, or had serum potassium $\geq 5.0 \mathrm{mmol} / \mathrm{L}$ at time of screening. Adolescents with type $1 \mathrm{DM}$ met inclusion criteria if they were $\geq 12$ and $\leq 18$ years old, had BMI $18-25 \mathrm{~kg} / \mathrm{m}^{2}$, and had HbAlc $<9.0 \%$ at time of screening. Adolescents were excluded if they were current smokers or had quit smoking $<6$ months prior, were taking vasoactive medications, had LDL cholesterol $\geq 160 \mathrm{mg} / \mathrm{dL}$, had blood pressure $<100 / 60 \mathrm{~mm} \mathrm{Hg}$, had pulse oximetry $<90 \%$, were pregnant or nursing, had history of cardiovascular, peripheral vascular, or liver disease, or had history of ketoacidosis within the previous 12 calendar months. Stringent eligibility criteria were used to avoid confounding influence of these factors (ie, smoking, chronic severe hyperglycemia, hyperlipidemia, renal dysfunction) on endothelial function and acute vascular changes assessed in other study protocols. Baseline data drawn from these clinical studies provided data for this cross-sectional study.

\section{Study design}

We followed the Strengthening the Reporting of Observational Studies in Epidemiology guidelines ${ }^{17}$ to analyze and report this cross-sectional study. We analyzed baseline measures of cfPWV, AIx, FMD, systolic blood pressure (SBP), diastolic blood pressure (DBP), mean arterial pressure (MAP), and heart rate for each participant. All vascular assessments in this study were measured per expert recommendations ${ }^{18}{ }^{19}$ by the same trained operator. Study participants were instructed to avoid alcohol, exercise, and caffeine for 24 hours and to fast overnight prior to admission to the CRU.

\section{Vascular measures}

Hemodynamic function

Clinical hemodynamic assessments were obtained at the initial screening visit. Blood pressure and heart rate were obtained with a GE Dinamap ProCare 400 vital signs monitor (GE Healthcare; Chicago, Illinois, USA). MAP was calculated as: $\mathrm{DBP}+((1 / 3) \times(\mathrm{SBP}-\mathrm{DBP})){ }^{20}$

\section{Carotid-femoral pulse wave velocity}

cfPWV was measured with a SphygmoCor tonometer (AtCor Medical; Naperville, Illinois, USA) to assess central aortic stiffness. To minimize the effects of sympathetic activity on cfPWV measurements, participants rested supine in a temperature-controlled room for at least $15 \mathrm{~min}$ prior to measurement. We measured the distance from the suprasternal notch to the carotid pulse and from the suprasternal notch to the ipsilateral 
femoral pulse. For each cfPWV measure, $10 \mathrm{~s}$ of carotid and $10 \mathrm{~s}$ of femoral arterial waveforms were recorded. cfPWV measures were made in duplicate and the mean value was reported. cfPWV intraobserver reliability was also assessed by having the operator record three serial cfPWV measurements on the same subject over a 4-hour period. The coefficient of variation for cfPWV was 3.63\%, indicating good intraobserver reliability. ${ }^{21}$

\section{Augmentation index}

To assess muscular conduit arterial stiffness, we measured AIx non-invasively with a SphygmoCor tonometer. AIx measurements were obtained at the radial artery by the same trained operator with participants lying in the supine position in a temperature-controlled room for at least $15 \mathrm{~min}$ prior to measurement. AIx was calculated as the difference of the amplitude of the late systolic peak to the early systolic peak divided by the pulse pressure and expressed as a percentage. AIx values were determined for each pulse over a $30 \mathrm{~s}$ period, and a mean value was calculated by the device for each patient and corrected for a heart rate of 75 beats/min.

\section{Flow-mediated dilation}

We measured left brachial artery FMD with the EPIQ 7 cardiovascular ultrasound (Philips Medical Systems; Andover, Massachusetts, USA) instrument with a linear array probe (L12-3) steadied by a probe-holder as described previously. ${ }^{22}$ FMD images were analyzed using Brachial Analyzer (Medical Imaging Applications; Coralville, Iowa, USA) edge detection software by study personnel blinded to subject. We assessed FMD intraobserver reliability by having the same trained observer record eight serial FMD measurements on the same subject over a 4-hour period. The coefficient of variation was $7.41 \%$, indicating good intraobserver reliability. ${ }^{19} 21$

\section{Biochemical analyses}

Complete blood count, comprehensive metabolic panel, lipid panel, HbAlc, and serum pregnancy tests were assayed at the UVA Clinical Chemistry Laboratory.

\section{Statistical analyses}

\section{Sample size}

The collective study cohort included participants from two separate studies of adolescents and adults with type 1 DM. All persons with type 1 DM from these prior studies were included in the current study.

\section{Statistical methods}

A total of two multivariable ordinary least squares (OLS) regression models were constructed to examine the multivariable relationship between cfPWV and potential predictors. The first OLS regression model examined the linear relationships between cfPWV $\left(\log _{\mathrm{e}}\right)$ and the multivariable predictor demographic dataset of patient age (years), duration of DM (years), and HbAlc (\%). The second OLS regression model examined the linear relationships between cfPWV $\left(\log _{\mathrm{e}}\right)$ and the vascular measures multivariable predictor dataset of AIx (\%), MAP ( $\mathrm{mm} \mathrm{Hg}$ ), FMD (\% change), and heart rate (beats/ min). The OLS regression model type III extra-sum of squares F-tests served as the pivotal quantities for testing the null hypothesis that cfPWV $\left(\log _{\mathrm{e}}\right)$ was not uniquely associated with the predictor variable after accounting for the variability in $\mathrm{cfPWV}\left(\log _{\mathrm{e}}\right)$ that was explained by the remaining set of OLS regression model predictors. A $\mathrm{p} \leq 0.05$ decision rule served as the null hypothesis rejection rule.

\section{RESULTS}

Demographic and baseline vascular data from the 41 participants are presented in table 1 and separated by sex. Sixteen adolescents (39\%) and 25 adults (61\%) were included. The cohort was $34 \%$ female, had mean age of 24 years, mean DM duration of 14 years, and mean HbAlc of $7.9 \%$. Male and female participants were similar in age, DM duration, HbAlc, blood pressure, BMI, and lipid profile. Male participants as a group were taller, had lower per cent body fat, and higher $\mathrm{VO}_{2 \max }$ compared with female participants. Regarding vascular measures, AIx and FMD were not significantly different between sexes but cfPWV was higher in male participants compared with female participants (indicating greater central aortic stiffness in male participants).

We examined macrovascular function based on duration of DM. Defining arterial stiffness as cfPWV $>90$ th percentile for sex and age based on previously defined reference intervals, ${ }^{23} 1$ of $6(16.7 \%)$ participants with DM duration $<5$ years, 3 of 7 (42.8\%) participants with DM duration 5-10 years, and 2 of $23(8.7 \%)$ participants with DM duration $>10$ years met criteria for arterial stiffness. Five participants in the DM duration 5-10 years group had cfPWV measures of insufficient quality for analysis. Conversely, there was a higher frequency of NO-dependent endothelial dysfunction (defined as FMD $\leq 8.1 \%^{2425}$ ) among our study cohort. Specifically, 5 of $6(83.3 \%)$ participants with DM duration $<5$ years, 7 of $12(58.3 \%)$ participants with DM duration 5-10 years, and 14 of 23 (60.9\%) participants with DM duration $>10$ years met criteria for impaired FMD. Four participants (2 males/2 females) had elevated urine albumin-to-creatinine ratio (ie, $>30 \mathrm{mg} / \mathrm{g}$ ) on screening. All of these participants with microalbuminuria on screening demonstrated impaired endothelial function and one met criteria for arterial stiffness.

Six participants were receiving antihypertension treatment, each with an ACE inhibitor, but this was not significantly different between sexes. Characteristics of these participants are included in the online supplemental table. Five of six had impaired FMD and one had evidence of central arterial stiffness.

The demographic OLS regression model is presented in table 2. Sex and age provided unique predictive information about cfPWV, while DM duration and HbAlc did 
Table 1 Baseline characteristics by sex

\begin{tabular}{|c|c|c|c|}
\hline & Combined $(n=41)$ & Female participants $(n=14)$ & Male participants $(n=27)$ \\
\hline Age (years) & $24.2 \pm 1.6$ & $24 \pm 2.8$ & $24.3 \pm 2$ \\
\hline Ethnicity (n (\%)) & $\begin{array}{l}\text { Non-Hispanic } \\
\text { White: } 37(90.2) \\
\text { Black: } 4 \text { (9.8) }\end{array}$ & $\begin{array}{l}\text { Non-Hispanic } \\
\text { White: } 13(92.9) \\
\text { Black: } 1 \text { (7.1) }\end{array}$ & $\begin{array}{l}\text { Non-Hispanic } \\
\text { White: } 24 \text { (88.9) } \\
\text { Black: } 3 \text { (11.1) }\end{array}$ \\
\hline DM duration (years) & $14.5 \pm 1.6$ & $14.9 \pm 2.5$ & $14.3 \pm 2.1$ \\
\hline $\mathrm{HbA} 1 \mathrm{c}(\% / \mathrm{mmol} / \mathrm{mol})$ & $7.9 \pm 0.2 / 63$ & $7.7 \pm 0.4 / 61$ & $8.0 \pm 0.2 / 64$ \\
\hline Urine albumin-to-creatinine ratio $(\mathrm{mg} / \mathrm{g})$ & $14.5 \pm 3.2$ & $14.9 \pm 6.1$ & $14.3 \pm 3.8$ \\
\hline Use of antihypertensive medication (n (\%)) & $6(14.6 \%)$ & $1(7 \%)$ & $5(18.5 \%)$ \\
\hline $\mathrm{BMI}\left(\mathrm{kg} / \mathrm{m}^{2}\right)$ & $24.1 \pm 0.6$ & $24.6 \pm 0.8$ & $23.9 \pm 0.8$ \\
\hline Height (cm) & $173.6 \pm 1.5$ & $167 \pm 1.3$ & $177.1 \pm 1.9^{*}$ \\
\hline Weight (kg) & $73.6 \pm 2.5$ & $68.4 \pm 2.9$ & $76.3 \pm 3.4^{*}$ \\
\hline Per cent body fat (\%) & $22.7 \pm 1.5$ & $29.7 \pm 2$ & $19 \pm 1.6^{*}$ \\
\hline $\mathrm{VO}_{2 \max }(\mathrm{mL} / \mathrm{kg} / \mathrm{min})$ & $40.7 \pm 1.6$ & $32.8 \pm 1.3$ & $44.9 \pm 1.8^{\star}$ \\
\hline Systolic blood pressure $(\mathrm{mm} \mathrm{Hg})$ & $119.8 \pm 2.7$ & $112.9 \pm 4$ & $123.3 \pm 3.3$ \\
\hline Diastolic blood pressure $(\mathrm{mm} \mathrm{Hg})$ & $68.8 \pm 1.4$ & $67.6 \pm 2.2$ & $69.4 \pm 1.8$ \\
\hline Total cholesterol (mg/dL/mmol/L) & $160.4 \pm 5.4 / 4.15 \pm 0.1$ & $172.4 \pm 8.9 / 4.46 \pm 0.2$ & $154.2 \pm 6.6 / 3.99 \pm 0.2$ \\
\hline LDL cholesterol (mg/dL/mmol/L) & $98.1 \pm 4.8 / 2.5 \pm 0.1$ & $107.6 \pm 7.4 / 2.8 \pm 0.2$ & $93.2 \pm 6.1 / 2.4 \pm 0.2$ \\
\hline $\mathrm{HDL}$ cholesterol (mg/dL/mmol/L) & $51.8 \pm 1.3 / 1.3$ & $54.4 \pm 1.9 / 1.4$ & $50.4 \pm 1.7 / 1.3$ \\
\hline Triglycerides (mg/dL/mmol/L) & $63.1 \pm 4.6 / 0.7 \pm 0.1$ & $62.5 \pm 6.6 / 0.8 \pm 0.1$ & $63.4 \pm 6.2 / 0.7+0.1$ \\
\hline FMD (\% change) & $7.4 \pm 0.4$ & $8.2 \pm 0.8$ & $7 \pm 0.5$ \\
\hline Alx (\%) & $3.8 \pm 2.1$ & $9.1 \pm 4.4$ & $1.3 \pm 2.2$ \\
\hline $\operatorname{cfPWV}(\mathrm{m} / \mathrm{s})$ & $6 \pm 0.3$ & $5.3 \pm 0.3$ & $6.4 \pm 0.4^{\star \star}$ \\
\hline
\end{tabular}

Data presented as mean \pm SEM. $P$ values represent Welch's t-tests comparing sexes.

${ }^{*} \mathrm{P}<0.001,{ }^{* *} \mathrm{p}=0.03$.

Alx, augmentation index; BMI, body mass index; cfPWV, carotid-femoral pulse wave velocity; DM, diabetes mellitus; FMD, flow-mediated dilation; HbA1c, hemoglobin A1c; HDL, high-density lipoprotein; LDL, low-density lipoprotein; $\mathrm{VO}_{2 \max }$, maximum oxygen uptake.

not. All four variables as a collective unit provided significant predictive information about cfPWV.

The vascular OLS regression model is presented in table 3. In this model, FMD alone offered unique predictive information about cfPWV, while AIx, MAP, and HR did not. All variables as a collective unit did not provide significant predictive information.

\section{DISCUSSION}

Our cohort with type $1 \mathrm{DM}$ exhibited a high proportion of NO-dependent endothelial dysfunction $(58 \%-83 \%$ of participants depending on diabetes duration group) and less pronounced but still noteworthy amount of central arterial stiffness $(8.7 \%-42.8 \%$ with cfPWV $>90$ th percentile based on age and gender). The fact that cfPWV was elevated to $>90$ th percentile in a moderate percentage of our study participants is in line with the prior, larger SEARCH CVD trial, which showed increased cfPWV in adolescents and young adults with type $1 \mathrm{DM}$ compared with healthy controls, although sex-based and genderbased cfPWV percentiles were not reported in that study. ${ }^{9}$ It should be noted that a majority of our participants' cfPWV were in line with their peers, and this may reduce our ability to detect meaningful predictive relationships with cfPWV. Impaired vasodilation measured by FMD is commonly seen early in the disease process of persons with type $1 \mathrm{DM}$, as others have reported $\sim 36 \%$ of children and adolescents already have reduced FMD within 5 years of diagnosis. ${ }^{24}{ }^{26}$ Despite reasonable glycemic control at the time of study, our population had an even greater prevalence of NO-dependent endothelial dysfunction that was relatively stable across groups with longer DM duration. The consistent finding of early impaired NO-dependent endothelial function and a modest association between FMD and early HbA1c in other studies have led some to hypothesize that there is a metabolic memory phenomenon in type $1 \mathrm{DM}$ whereby early glycemic control is highly formative of endothelial function, ${ }^{24}$ although more data are needed to validate this hypothesis. This may also explain why we saw no significant relationship between HbAlc at time of study and cfPWV.

Despite similar baseline characteristics and cardiovascular risk factors between sexes, men with type $1 \mathrm{DM}$ had higher cfPWV (indicating greater central arterial stiffness) compared with women with type $1 \mathrm{DM}$. This was despite male participants having higher $\mathrm{VO}_{2 \max }$ (suggesting greater cardiorespiratory fitness/muscle mass). Prior studies in other populations have shown 
Table 2 Demographic multivariable ordinary least squares regression model for cfPWV

\begin{tabular}{|c|c|c|c|c|c|}
\hline \multicolumn{6}{|c|}{ A: OLS regression coefficients for the regression of In(PWV) onto sex, age, duration of DM, and HbA1c } \\
\hline $\begin{array}{l}\text { Regression } \\
\text { parameter }\end{array}$ & $\begin{array}{l}\text { Parameter } \\
\text { estimate }\end{array}$ & SE & $\begin{array}{l}\text { Lower 95\% } \\
\text { CL }\end{array}$ & $\begin{array}{l}\text { Upper 95\% } \\
\text { CL }\end{array}$ & \\
\hline$\beta_{0}$ & 0.9896 & 0.3014 & 0.3749 & 1.6043 & \\
\hline$\beta_{1}$ & -0.1435 & 0.0681 & -0.2824 & -0.0046 & \\
\hline$\beta_{2}$ & 0.0190 & 0.0074 & 0.0039 & 0.0341 & \\
\hline$\beta_{3}$ & -0.0051 & 0.0073 & -0.02 & 0.0098 & \\
\hline$\beta_{4}$ & 0.0545 & 0.0299 & -0.0065 & 0.1155 & \\
\hline \multicolumn{6}{|c|}{ B: Type III ANOVA F-tests } \\
\hline Predictor variable & df & $\begin{array}{l}\text { Partial sum of } \\
\text { squares }\end{array}$ & $\begin{array}{l}\text { Mean square } \\
\text { error }\end{array}$ & F-statistic & P value \\
\hline Sex & 1 & 0.16 & 0.16 & 4.44 & 0.043 \\
\hline Age & 1 & 0.24 & 0.24 & 6.57 & 0.015 \\
\hline Duration DM & 1 & 0.02 & 0.02 & 0.49 & 0.489 \\
\hline $\mathrm{HbA1c}$ & 1 & 0.12 & 0.12 & 3.32 & 0.078 \\
\hline Total & 4 & 0.95 & 0.24 & 6.46 & 0.001 \\
\hline Error & 31 & 1.14 & 0.04 & & \\
\hline
\end{tabular}

(A) presents OLS regression coefficients for the OLS regression model: $E(\ln (\mathrm{cfPWV}) \mid \mathrm{X})=\beta_{0}+\beta_{1}(\mathrm{female})+\beta_{2}(\mathrm{age})+\beta_{3}(\mathrm{duration}$ of $\mathrm{DM})+\beta_{4}(\mathrm{HbA} 1 \mathrm{c})$. (B) presents type III ANOVA F-tests of the OLS analysis.

ANOVA, analysis of variance; cfPWV, carotid-femoral pulse wave velocity; DM, diabetes mellitus; HbA1c, hemoglobin A1c; OLS, ordinary least squares.

an association between higher cardiorespiratory fitness and lower $\mathrm{cfPWV}^{27}$ and that aerobic exercise training reduces $\mathrm{cfPWV}^{28}$ Furthermore, while women typically have lower cfPWV compared with age-matched men, the sex difference observed in our study was more exaggerated than that observed in healthy individuals. ${ }^{23}$ More male participants were treated for hypertension in our cohort; however, this was not statically different between

Table 3 Vascular multivariable ordinary least squares regression model for cfPWV

\begin{tabular}{|c|c|c|c|c|c|}
\hline \multicolumn{6}{|c|}{ A: OLS regression coefficients for the regression of In(cfPWV) onto Alx, MAP, FMD, and HR } \\
\hline Regression parameter & $\begin{array}{l}\text { Parameter } \\
\text { estimate }\end{array}$ & SE & $\begin{array}{l}\text { Lower 95\% } \\
\text { CL }\end{array}$ & $\begin{array}{l}\text { Upper 95\% } \\
\text { CL }\end{array}$ & \\
\hline$\beta_{1}$ & 0.0018 & 0.003 & -0.0043 & 0.0079 & \\
\hline$\beta_{2}$ & 0.0044 & 0.0038 & -0.0034 & 0.0122 & \\
\hline$\beta_{4}$ & -0.0034 & 0.0027 & -0.0089 & 0.0021 & \\
\hline \multicolumn{6}{|l|}{ B: Type III ANOVA F-tests } \\
\hline Predictor variable & df & Partial sum of squares & $\begin{array}{l}\text { Mean square } \\
\text { error }\end{array}$ & F-statistic & $P$ value \\
\hline Alx & 1 & 0.02 & 0.02 & 0.33 & 0.567 \\
\hline Total & 4 & 0.51 & 0.13 & 2.49 & 0.064 \\
\hline Error & 31 & 1.59 & 0.05 & & \\
\hline
\end{tabular}

(A) presents OLS regression coefficients for the OLS regression model: $E(\ln (\mathrm{cfPWV}) \mid \mathrm{X})=\beta_{0}+\beta_{1}(\mathrm{Alx})+\beta_{2}(\mathrm{MAP})+\beta_{3}(\mathrm{FMD})+\beta_{4}(\mathrm{HR})$. (B) present type III ANOVA F-tests of the OLS analysis.

Alx, augmentation index; ANOVA, analysis of variance; cfPWV, carotid-femoral pulse wave velocity; FMD, flow-mediated dilation; MAP, mean arterial pressure; OLS, ordinary least squares. 
sexes and those with hypertension were not disproportionately impacted by elevated cfPWV. Interestingly, there was no sex difference in either FMD or AIx. While larger studies in the type $1 \mathrm{DM}$ population are certainly needed to verify these findings, our initial results are hypothesis-generating. Recent evidence has exposed a twofold excess fatal CVD risk in women with type 1 DM, compared with their male counterparts, ${ }^{29}$ although reasons for this are unclear. In the current study, macrovascular function was comparable and aortic stiffness lower in women compared with men. These findings suggest that the increased CVD risk for women with type 1 DM may not be due to differences in macrovascular function. Coronary microvascular dysfunction is increasingly recognized as an important risk factor for CVD events $^{30}$ and is more prevalent in individuals with DM. ${ }^{31}$ Haas et al recently showed that women with type $2 \mathrm{DM}$ and good cardiometabolic control had reduced coronary microvascular function compared with men with type 2 DM. ${ }^{32}$ Whether such a sex-based discrepancy in coronary microvascular function exists in type $1 \mathrm{DM}$, with women potentially having greater dysfunction and this being a primary driver for their increased cardiovascular risk, warrants further investigation.

Contrary to our hypothesis, only age and sex provided unique predictive information about cfPWV in the demographics model. Interestingly, DM duration and HbAlc were not significant predictors. Age and sex are both known to impact cfPWV ${ }^{23}$ and we therefore included these variables in the demographics model to account for their expected influence. One prior study of adolescents with poorly controlled type $1 \mathrm{DM}$ did find a significant independent association between HbAlc and cfPWV, but as in the current study, DM duration was not significantly associated with cfPWV. ${ }^{33}$ The discordant results for HbAlc likely relate to the fact that their study had serial HbAlc measurements available for analysis, while ours included HbAlc measurement at only one time point prior to vascular measurements.

cfPWV is the gold standard estimate of arterial stiffness in adolescents and adults ${ }^{345}$ and has a strong independent association with subclinical atherosclerosis. ${ }^{35}$ As previously noted, cfPWV is an important risk marker for renal outcomes, cardiovascular events, and mortality in people with type $1 \mathrm{DM},{ }^{13}$ although few prior studies have examined predictors of cfPWV in this population. A multivariable regression model from the larger SEARCH CVD study with a population of 402 adolescents and young adults with type $1 \mathrm{DM}$ and 206 healthy controls found that factors uniquely associated with higher cfPWV included presence of type $1 \mathrm{DM}$, older age, race other than nonHispanic white, higher MAP, higher waist-to-height ratio, and presence of microalbuminuria. ${ }^{9}$ Another study of 68 individuals with type $1 \mathrm{DM}$ and 68 age-matched and sex-matched healthy controls found that age, BMI, type $1 \mathrm{DM}$, and low-grade inflammation predicted cfPWV in men, whereas age, BMI, MAP, and type $1 \mathrm{DM}$ predicted cfPWV in women. ${ }^{6}$ Within our vascular model, we found that FMD significantly predicted cfPWV, while HR, MAP, and AIx did not. We were surprised that MAP did not have a significant association with cfPWV in light of the prior results in type $1 \mathrm{DM}$ and the fact that MAP is a known cfPWV determinant. ${ }^{18}$ This lack of association likely relates to the fact that the blood pressure used to calculate MAP was obtained at the initial screening visit (up to 4 weeks prior to vascular studies). We used MAP obtained at this time, instead of study admission, to assess the reliability of MAP measured in a clinical environment (eg, an ambulatory office setting) to predict arterial stiffness, and a change in blood pressures/MAP across this time is possible.

We also did not observe a significant association between AIx and cfPWV. In type 1 DM, both AIx and cfPWV are elevated ${ }^{36}$ and predict cardiovascular events and mortality. ${ }^{1213}$ The lack of association we observed may relate to intrinsic differences between AIx and cfPWV as measures of vascular stiffness. Whereas AIx is a peripheral arterial measurement influenced by numerous determinants including heart rate and contractility, cfPWV is a more direct measurement of central aortic and aortoiliac artery stiffness, and cfPWV is considered the 'goldstandard' non-invasive measure of arterial stiffness. ${ }^{37} \mathrm{In}$ young healthy individuals, central arteries show greater elasticity compared with more muscular peripheral arteries, and central arteries are preferentially stiffened by aging and hypertension. ${ }^{38}$ In type $1 \mathrm{DM}$, a dissociation between central and peripheral arterial stiffness, as evaluated by cfPWV and AIx, was also previously reported with a lack of association between the two measures after adjusting for cardiovascular risk factors. ${ }^{39}$ Taking into account our findings, this may suggest a more important role for cfPWV measurement in early cardiovascular risk stratification for type $1 \mathrm{DM}$.

That only FMD provided unique predictive information about cfPWV was surprising given that FMD measures brachial artery NO-dependent endothelial function. The endothelium releases the potent vasodilator NO in response to shear stress induced by reactive hyperemia elicited by $\mathrm{FMD}^{40}$ testing. NO bioavailability influences dynamic changes in the arterial wall, ${ }^{41}$ and preserved endothelial NO production is an important atheroprotective factor. FMD specifically has a strong inverse linear relationship with cardiovascular events and mortality. In fact, every $1 \%$ increase in FMD correlates with a $9 \%$ reduction in cardiovascular events. ${ }^{42}$

To our knowledge, the current study is the first to investigate the specific association between cfPWV and FMD within type $1 \mathrm{DM}$. A prior study of 68 individuals with type $1 \mathrm{DM}$ and 68 sex-matched and age-matched healthy controls reported that endothelial dysfunction was more frequent in the type $1 \mathrm{DM}$ cohort but not associated with cfPWV after adjusting for potential confounders. ${ }^{43}$ However, the study used reactive hyperemia peripheral arterial tonometry (RH-PAT) to assess endothelial function and it is noteworthy that RH-PAT failed to significantly correlate with FMD in either the Framingham Heart 
Study's Offspring, Third Generation, or Omni cohorts. ${ }^{44}$ Moreover, brachial artery FMD is recognized as the gold standard for non-invasive assessment of endothelial function. ${ }^{45}$ While FMD was a significant predictor of cfPWV in our type $1 \mathrm{DM}$ population, associations between cfPWV and FMD in other populations are inconsistent. In newly diagnosed hypertensive individuals $(\mathrm{n}=189), \mathrm{FMD}$ had a significant independent association with carotid intimal thickness but not cfPWV. ${ }^{46}$ Similarly, in the Young Finns study ( $\mathrm{n}=1754$ ), FMD did not modulate the association between cardiovascular risk factors and cfPWV in young adults. ${ }^{47}$ Among individuals with type $2 \mathrm{DM}$, a significant independent association was found between brachialankle cfPWV and FMD but only in individuals with less advanced atherosclerosis (ie, lesser carotid intima-media thickness). ${ }^{48}$ Others found that FMD was inversely associated with, but not a unique predictor of, cfPWV in individuals with long-standing type $2 \mathrm{DM}$ and hypertension. ${ }^{49}$ Still, the association between cfPWV and FMD in our type $1 \mathrm{DM}$ population raises the possibility that dysglycemia or perhaps insulin resistance, even in the absence of other cardiovascular risk factors, increases the risk of both vascular endothelial and arterial wall dysfunction. Along these lines, a recent meta-analysis of 58 studies examining endothelial function in persons with type $1 \mathrm{DM}$ $(n=2322)$ and healthy controls $(n=1777)$ corroborated the presence of early endothelial and vascular smooth muscle dysfunction in children and adults with type 1 $\mathrm{DM}^{50}{ }^{50}$ The study also reported that endothelial dysfunction seems to be more pronounced within macrovascular than microvascular beds, fostering the debate on their relative temporal appearance.

While our study provides a unique look at macrovascular function in adolescents and adults with uncomplicated type $1 \mathrm{DM}$, it contains several important limitations. First, it is a small cross-sectional examination across a wide age spectrum. Second, it defines neither the time sequence nor the ontology of macrovascular dysfunction. Third, this study includes HbAlc at only one time point. Serial HbAlc measures would provide valuable data when evaluating long-term vascular function. Fourth, the restrictive eligibility criteria limits generalizability essentially to only individuals with type $1 \mathrm{DM}$ who are otherwise healthy and have not yet experienced diabetes-related complications. With these limitations in mind, the similar representation of cardiovascular risk factors between male and female participants in this cohort provides valuable information about early macrovascular function across sexes in type 1 DM prior to the advent of microvascular or macrovascular complications.

In conclusion, age, sex, and FMD were the only factors uniquely associated with $\mathrm{cfPWV}$ in our cohort of adolescents and adults with uncomplicated type $1 \mathrm{DM}$. Male participants had higher cfPWV, indicating greater central arterial stiffness, but similar FMD and AIx to female participants. Endothelial dysfunction was highly prevalent among our participants regardless of DM duration. Further study is warranted to determine the generalizability of our results and define the sequence of and sex differences in macrovascular dysfunction present in type $1 \mathrm{DM}$.

Contributors KML and WBH: study planning, data analysis, manuscript preparation; JTP: data analysis methods, data analysis, manuscript preparation; LAJ: subject recruitment, study execution, data analysis, and manuscript preparation; LMH: subject recruitment, study execution, manuscript preparation; EJB: study planning, execution, data analysis, manuscript preparation, and funding acquisition. KML acts as guarantor for the work.

Funding This work was supported by research grants from the NIH (DK101944 and DK073059) to EJB and in part by the National Center For Advancing Translational Sciences of the National Institutes of Health under Award Numbers KL2TR003016/ULTR003015 (to KML and WBH as iTHRIV scholars).

Disclaimer The content is solely the responsibility of the authors and does not necessarily represent the official views of the National Institutes of Health.

Competing interests None declared.

Patient consent for publication Not applicable.

Ethics approval The University of Virginia Health Science Research Institutional Review Board approved the clinical trials from which the data for this cohort study were obtained (IRB\#18237 and IRB\#15312). Each participant gave written informed consent at their initial visit in accordance with the Declaration of Helsinki before screening to verify inclusion/exclusion criteria.

Provenance and peer review Not commissioned; externally peer reviewed.

Data availability statement Data are available on reasonable request.

Supplemental material This content has been supplied by the author(s). It has not been vetted by BMJ Publishing Group Limited (BMJ) and may not have been peer-reviewed. Any opinions or recommendations discussed are solely those of the author(s) and are not endorsed by BMJ. BMJ disclaims all liability and responsibility arising from any reliance placed on the content. Where the content includes any translated material, BMJ does not warrant the accuracy and reliability of the translations (including but not limited to local regulations, clinical guidelines, terminology, drug names and drug dosages), and is not responsible for any error and/or omissions arising from translation and adaptation or otherwise.

Open access This is an open access article distributed in accordance with the Creative Commons Attribution Non Commercial (CC BY-NC 4.0) license, which permits others to distribute, remix, adapt, build upon this work non-commercially, and license their derivative works on different terms, provided the original work is properly cited, appropriate credit is given, any changes made indicated, and the use is non-commercial. See: http://creativecommons.org/licenses/by-nc/4.0/.

ORCID iD

Kaitlin M Love http://orcid.org/0000-0002-9352-393X

\section{REFERENCES}

1 Yu S, McEniery CM. Central versus peripheral artery stiffening and cardiovascular risk. Arterioscler Thromb Vasc Biol 2020;40:1028-33.

2 Zieman SJ, Melenovsky V, Kass DA. Mechanisms, pathophysiology, and therapy of arterial stiffness. Arterioscler Thromb Vasc Biol 2005;25:932-43.

3 Henry RMA, Kostense PJ, Spijkerman AMW, et al. Arterial stiffness increases with deteriorating glucose tolerance status: the Hoorn study. Circulation 2003;107:2089-95.

4 Sengstock DM, Vaitkevicius PV, Supiano MA. Arterial stiffness is related to insulin resistance in nondiabetic hypertensive older adults. J Clin Endocrinol Metab 2005;90:2823-7.

5 Gordin D, Saraheimo M, Tuomikangas J, et al. Influence of postprandial hyperglycemic conditions on arterial stiffness in patients with type 2 diabetes. J Clin Endocrinol Metab 2016;101:1134-43

6 Llaurado G, Ceperuelo-Mallafre V, Vilardell C, et al. Arterial stiffness is increased in patients with type 1 diabetes without cardiovascular disease: a potential role of low-grade inflammation. Diabetes Care 2012;35:1083-9.

7 George B, Bantwal G, Ayyar V, et al. Occurrence of increased arterial stiffness in a cohort of adult patients with type 1 diabetes mellitus when compared to normoglycemic controls. J Diabetes Sci Technol 2015;9:138-44. 
8 Zahner GJ, Gruendl MA, Spaulding KA, et al. Association between arterial stiffness and peripheral artery disease as measured by radial artery tonometry. J Vasc Surg 2017;66:1518-26.

9 Shah AS, Wadwa RP, Dabelea D, et al. Arterial stiffness in adolescents and young adults with and without type 1 diabetes: the search CVD study. Pediatr Diabetes 2015;16:367-74.

10 Bradley TJ, Slorach C, Mahmud FH, et al. Early changes in cardiovascular structure and function in adolescents with type 1 diabetes. Cardiovasc Diabetol 2016;15:31.

11 Heier M, Stensæth KH, Brunborg C, et al. Increased arterial stiffness in childhood onset diabetes: a cardiovascular magnetic resonance study. Eur Heart J Cardiovasc Imaging 2018;19:694-700.

12 Tynjälä A, Forsblom C, Harjutsalo V, et al. Arterial stiffness predicts mortality in individuals with type 1 diabetes. Diabetes Care 2020;43:2266-71.

13 Tougaard NH, Theilade S, Winther SA, et al. Carotid-Femoral pulse wave velocity as a risk marker for development of complications in type 1 diabetes mellitus. J Am Heart Assoc 2020;9:e017165.

14 Dabelea D, Talton JW, D'Agostino R, et al. Cardiovascular risk factors are associated with increased arterial stiffness in youth with type 1 diabetes: the search CVD study. Diabetes Care 2013;36:3938-43

15 Jones AG, Hattersley AT. The clinical utility of C-peptide measurement in the care of patients with diabetes. Diabet Med 2013;30:803-17.

16 Leighton E, Sainsbury CA, Jones GC. A practical review of Cpeptide testing in diabetes. Diabetes Ther 2017;8:475-87.

17 Dwan K, Li T, Altman DG, et al. Consort 2010 statement: extension to randomised crossover trials. BMJ 2019;366:14378.

18 Townsend RR, Wilkinson IB, Schiffrin EL, et al. Recommendations for improving and standardizing vascular research on arterial stiffness: a scientific statement from the American heart association. Hypertension 2015;66:698-722

19 Thijssen DHJ, Bruno RM, van Mil ACCM, et al. Expert consensus and evidence-based recommendations for the assessment of flowmediated dilation in humans. Eur Heart J 2019;40:2534-47.

20 DeMers D, Wachs D. Physiology, mean arterial pressure. Treasure Island (FL): StatPearls, 2021.

21 Shechtman O. The Coefficient of Variation as an Index of Measurement Reliability. In: SAR D, Williams GM, eds. Methods of clinical epidemiology. Berlin, Heidelberg: Springer Berlin Heidelberg, 2013: 39-49.

22 Jahn LA, Hartline L, Rao N, et al. Insulin enhances endothelial function throughout the arterial tree in healthy but not metabolic syndrome subjects. J Clin Endocrinol Metab 2016;101:1198-206.

23 Diaz A, Zócalo Y, Bia D, et al. Reference intervals and percentiles for carotid-femoral pulse wave velocity in a healthy population aged between 9 and 87 years. J Clin Hypertens 2018;20:659-71.

24 Cé GV, Rohde LE, da Silva AMV, et al. Endothelial dysfunction is related to poor glycemic control in adolescents with type 1 diabetes under 5 years of disease: evidence of metabolic memory. J Clin Endocrinol Metab 2011;96:1493-9.

25 Gokce N, Keaney JF, Hunter LM, et al. Predictive value of noninvasively determined endothelial dysfunction for long-term cardiovascular events in patients with peripheral vascular disease. $J$ Am Coll Cardiol 2003;41:1769-75.

26 Järvisalo MJ, Raitakari M, Toikka JO, et al. Endothelial dysfunction and increased arterial intima-media thickness in children with type 1 diabetes. Circulation 2004;109:1750-5

27 Deiseroth A, Streese L, Köchli S, et al. Exercise and arterial stiffness in the elderly: a combined cross-sectional and randomized controlled trial (EXAMIN age). Front Physiol 2019;10:1119.

28 Huang C, Wang J, Deng S, et al. The effects of aerobic endurance exercise on pulse wave velocity and intima media thickness in adults: a systematic review and meta-analysis. Scand J Med Sci Sports 2016;26:478-87.

29 Huxley RR, Peters SAE, Mishra GD, et al. Risk of all-cause mortality and vascular events in women versus men with type 1 diabetes: a systematic review and meta-analysis. The Lancet Diabetes Endocrinol 2015;3:198-206.

30 Herzog BA, Husmann L, Valenta I. Long-term prognostic value of $13 \mathrm{~N}$-ammonia myocardial perfusion positron emission tomography added value of coronary flow reserve. J Am Coll Cardiol 2009:54:150-6.

31 Di Carli MF, Janisse J, Ager J, Grunberger G, et al. Role of chronic hyperglycemia in the pathogenesis of coronary microvascular dysfunction in diabetes. J Am Coll Cardiol 2003;41:1387-93.

32 Haas AV, Rosner BA, Kwong RY, et al. Sex differences in coronary microvascular function in individuals with type 2 diabetes. Diabetes 2019;68:631-6.

33 Obermannova B, Petruzelkova L, Sulakova T, et al. Hba1C but not diabetes duration predicts increased arterial stiffness in adolescents with poorly controlled type 1 diabetes. Pediatr Diabetes 2017;18:304-10.

34 Urbina EM, Williams RV, Alpert BS. Noninvasive assessment of subclinical atherosclerosis in children and adolescents: recommendations for standard assessment for clinical research: a scientific statement from the American heart association. Hypertension 2009;54:919-50.

35 Blacher J, Asmar R, Djane S, et al. Aortic pulse wave velocity as a marker of cardiovascular risk in hypertensive patients. Hypertension 1999;33:1111-7.

36 Shah AS, Black S, Wadwa RP, et al. Insulin sensitivity and arterial stiffness in youth with type 1 diabetes: the search CVD study. J Diabetes Complications 2015;29:512-6.

37 Laurent S, Cockcroft J, Van Bortel L, et al. Expert consensus document on arterial stiffness: methodological issues and clinical applications. Eur Heart J 2006;27:2588-605.

38 Benetos A, Laurent S, Hoeks AP, et al. Arterial alterations with aging and high blood pressure. A noninvasive study of carotid and femoral arteries. Arterioscler Thromb 1993:13:90-7.

39 Llauradó G, Simó R, Villaplana M, et al. Can augmentation index substitute aortic pulse wave velocity in the assessment of central arterial stiffness in type 1 diabetes? Acta Diabetol 2012;49:253-7.

40 Tomiyama $\mathrm{H}$, Yamashina A. Non-invasive vascular function tests: their pathophysiological background and clinical application. Circ J 2010;74:24-33.

41 Vanhoutte PM, Shimokawa H, Feletou M, et al. Endothelial dysfunction and vascular disease - a 30th anniversary update. Acta Physiologica 2017;219:22-96.

42 Green DJ, Jones H, Thijssen D. Flow-mediated dilation and cardiovascular event prediction: does nitric oxide matter? Hypertension 2011;57:363-9.

43 Llauradó G, Ceperuelo-Mallafré V, Vilardell C, et al. Impaired endothelial function is not associated with arterial stiffness in adults with type 1 diabetes. Diabetes Metab 2013;39:355-62.

44 Hamburg NM, Palmisano J, Larson MG. Relation of brachial and digital measures of vascular function in the community: the Framingham heart study. Hypertension 2011;57:390-6.

45 Stoner L, Tarrant MA, Fryer S, et al. How should flow-mediated dilation be normalized to its stimulus? Clin Physiol Funct Imaging 2013;33:75-8.

46 Tatasciore A, Di Nicola M, Tommasi R, et al. From short-term blood pressure variability to atherosclerosis: relative roles of vascular stiffness and endothelial dysfunction. J Clin Hypertens 2020;22:1218-27.

47 Koivistoinen T, Virtanen M, Hutri-Kähönen N, et al. Arterial pulse wave velocity in relation to carotid intima-media thickness, brachial flow-mediated dilation and carotid artery distensibility: the cardiovascular risk in young finns study and the health 2000 survey. Atherosclerosis 2012;220:387-93.

48 Yokoyama H, Sone H, Saito K. Flow-mediated dilation is associated with microalbuminuria independent of cardiovascular risk factors in type 2 diabetes - interrelations with arterial thickness and stiffness. $J$ Atheroscler Thromb 2011:18:744-52.

49 Naka KK, Papathanassiou K, Bechlioulis A, et al. Determinants of vascular function in patients with type 2 diabetes. Cardiovasc Diabetol 2012;11:127.

50 Lespagnol E, Dauchet L, Pawlak-Chaouch M, et al. Early endothelial dysfunction in type 1 diabetes is accompanied by an impairment of vascular smooth muscle function: a meta-analysis. Front Endocrinol 2020;11:203. 